\title{
EL GOBIERNO CORPORATIVO EN EL ÁMBITO DEL SECTOR PÚBLICO: UN ESTUDIO BIBLIOMÉTRICO EN LAS REVISTAS UBICADAS EN EL ÁREA DE ADMINISTRACIÓN PÚBLICA*
}

\author{
JESÚS MAURICIO FLÓREZ-PARRA* \\ UNIVERSIDAD DE GRANADA (ESPAÑA) \\ Recibido/ Received/ Recebido: 27/08/2015-Aceptado/ Accepted / Aprovado: 21/06/2016
}

\section{Resumen}

Esta investigación se centra en un estudio bibliométrico comprendido entre los años 2007-2015. Se analizan las temáticas más relevantes y significativas, las universidades que investigan el fenómeno del gobierno corporativo (GC) en el ámbito público y las revistas que abordan esta temática. Para este fin, se tomaron las 47 revistas de mayor prestigio e impacto internacional incluidas en Public Administration. El análisis de los resultados pone sobre el relieve la implementación del GC en el sector público en áreas como la educación, la sanidad o los gobiernos locales y el predominio que ejerce la cultura anglosajona a través de las diversas revistas académicas del Journal Citation Reports.

Palabras clave: Gobierno corporativo; Sector público; Revistas internacionales; Bibliometría; Transparencia.

\section{CORPORATE GOVERNANCE IN THE FIELD OF PUBLIC SECTOR: A BIBLIOMETRIC STUDY IN MAGAZINES LOCATED IN THE AREA OF PUBLIC ADMINISTRATION}

\begin{abstract}
This research focuses on a bibliometric study comprised between 2007-2015. The most relevant and significant themes are discussed, universities investigating the phenomenon of corporate governance (CG) in the public domain and magazines that address this issue. To this end 47 of the most prestigious journals and with international impact included in Public Administration were taken. The analysis of the results put on the relief CG implementation in the public sector in areas such as education, health and local government and the dominance exerted by the Anglo-Saxon culture through various academic journals of The Journal Citation Reports.

Keywords: Corporate governance; Public sector; International journals; Bibliometrics; Transparency.
\end{abstract}

Artículo de revisión.

Doctor en Ciencias Económicas y Empresariales por la Universidad de Granada-España. Adscrito como Profesor de la Facultad de Ciencias Económicas, Administrativas y Contables, Programa Académico de Contaduría Pública de la Fundación Universidad Autónoma de Colombia (FUAC): Dirección postal: Calle comandante Cousteau № 2 Maracena-Granada- España. Código postal 18200. Teléfono: (34) 626842081. Correo electrónico: jlorez@correo.ugr.es 


\title{
O GOVERNO CORPORATIVO NO ÂMBITO DO SETOR PÚBLICO: UM ESTUDO BIBLIOMÉTRICO NAS REVISTAS LOCALIZADAS NA ÁREA DE ADMINISTRAÇÃO PÚBLICA
}

\begin{abstract}
Resumo
Esta pesquisa se concentra num estudo bibliométrico realizado entre os anos 2007-2015. Analisam-se as temáticas mais relevantes e significativas, as universidades que pesquisam o fenômeno do governo corporativo (GC) no âmbito público e as revistas que abordam esta temática. Para este fim, tomaram-se as 47 revistas de maior prestígio e impacto internacional incluídas em Public Administration. A análise dos resultados destaca a implementação do GC no setor público em áreas como a educação, a previdência ou os governos locais e o predomínio que exerce a cultura anglo-saxã através das diversas revistas acadêmicas do Journal Citation Reports.

Palavras chave: Governo corporativo; Setor público; Revistas internacionais; Bibliometria; Transparência.
\end{abstract}

Flórez-Parra, J. (2017). El Gobierno Corporativo en el ámbito del sector público: un estudio bibliométrico en las revistas ubicadas en el área de Administración Pública. En: Revista de la Facultad de Ciencias Económica: Investigación y Reflexión. rev.fac.cienc.econ, XXV (1). DOI: http://dx.doi.org/10.18359/rfce.743

JEL: G34, G39, O16.

\section{Introducción}

A través de la historia el gobierno corporativo (GC) ha venido evolucionando a tal punto que algunos investigadores como Gómez ^ Korine (2005) han clasificado al GC en tres grandes periodos: la primera etapa, data de la revolución industrial hasta 1920, en estos años el GC ha girado sobre el modelo de gestión que tiene como predomino a la familia fundadora; entre 1920-1970, su énfasis se establece en la gestión que tiene el gerente y finalmente una tercera fase, que arranca desde la década de los 70 en adelante, su característica principal es que la gestión es destinada a una mayor rendición de cuentas a la sociedad.

Podemos decir que el GC ha sido promovido principalmente por la cultura anglosajona, su impacto ha transcendido a nivel mundial y ha llegado a otras culturas como la de los países de Europa Continental, los Países Latinos e incluso Japón. Estas cultu- ras han venido incorporando su concepto y su forma de gestión a las estructuras empresariales con la intención de mejorar su eficiencia y eficacia en aras de una mayor competitividad (Weimer \& Paper, 1999). En gran medida el GC viene teniendo una relevancia en el contexto internacional y su influencia se pone de manifiesto con la elaboración, por diversos países, de códigos de buenas prácticas (Haxhi, Ees \& Sorge, 2013; Cuomo, Mallin \& Zattoni, 2016). Los códigos se establecen con el objetivo de crear normas uniformes de buen gobierno basado en la autorregulación (Hooghiemstra \& Van Ees, 2011).

Básicamente el GC ha tenido diversas corrientes teóricas, pero en la literatura existe un predominio de dos postulados que son comúnmente mencionados y según Cuervo (2002) los identifica con la teoría de la agencia, su característica principal es la de delegar la autoridad a un director o gerente para tomar decisiones (Jensen \& Mecklingn, 1976), y la 


\section{EL GOBIERNO CORPORATIVO EN EL ÁMBITO DEL SECTOR PÚBLICO: UN ESTUDIO BIBLIOMÉTRICO EN LAS REVISTAS UBICADAS EN EL ÁREA DE ADMINISTRACIÓN PÚBLICA}

teoría de los stakeholders, que consiste en que un grupo de personas (accionistas, acreedores, trabajadores entre otros) pueden verse afectados por las actividades y la toma de decisiones de la organización (Freeman, 1984, 1994; Donaldson \& Preston, 1995).

Otros autores como Carver (2010) identifica la teoría global de la gobernanza, esta trata de las prácticas y las relaciones entre los consejos de administración y la necesidad de establecer una rendición de cuentas entre los propietarios y los operadores. Ho (2005) establece una detallada clasificación de varios modelos teóricos del GC como son: la teoría de los stewardship, esta plantea que los ejecutivos estén motivados para actuar con mayor interés con sus gerentes o directores para lograr los objetivos de la organización (Donaldson, \& Davis, 1991; Davis, Schoorman \& Donaldson 1997); modelo político, consiste en la implantación de estrategias para recabar votos entre los accionistas, con la intención de establecer políticas corporativas; modelo participativo, este trata sobre la forma cómo el GC debe adaptarse a los nuevos cambios; modelo de liderazgo estratégico, este aborda el apoyo de la junta directiva en la realización de tareas que fomenten mejoras para la empresa y finalmente el modelo de política de gobierno, este consiste en comunicar al personal las expectativas a cumplir así como los parámetros y procesos en las tomas de las decisiones.

El GC no solo se ha extendido a nivel teórico o global si no también se ha trasmitido a otros campos del conocimiento como pueden ser la economía, las finanzas, la gestión, el derecho y la contabilidad (Durisin \& Puzone, 2009). El campo del sector público no ha sido ajeno a estos cambios y un ejemplo de ello es la incorporación de diversos aspectos del GC en el ámbito de la educación, en especial la universitaria (Flórez, 2013, Flórez, López \& López, 2014a, Christopher, 2015; Lokuwaduge \& Armstrong, 2015).

La conceptualización más común y generalmente aceptada sobre el GC se refiere al sistema en el cual las empresas son dirigidas y controladas (Cadbury, 1992). Su concepto se puede aplicar tanto en el ámbito privado como en el público. El GC también es descrito como la manera de controlar las actividades de la compañía (Hardman, 1996); es considerado como una herramienta de vigilancia (La Porta, Lopez-De-Silanes, Shleifer \& Vishny, 2000); es un marco legal, institucional y cultural (Weimer \& Pape, 1999); es un sistema de contrato que define las relaciones entre los tres agentes principales en la corporación: el soberano, el gobernado y el gobierno (Gómez \& Korine, 2005); es una forma de relación existente entre los diversos stakeholders (Luan \& Tang, 2007); es un conjunto amplio de información financiera y de mecanismos de control (Carnegie \& Tuck, 2010); es una estructura que proporciona derechos y responsabilidades entre los diversos participantes en la sociedad (Wellens \& Jegers, 2011); es una herramienta que integra la responsabilidad y la transparencia en las diversas áreas de la dirección estratégica y la planificación (Stewart, Asha, Shulman \& Ng, 2012); es un conjunto de mecanismos que influyen en la toma de las decisiones de los consejos de administración y sus distintos comités (Adut, Holder \& Robin, 2013).

La estructura del GC suele estar formada por un consejo de administración, por comités específicos, directores (director general o alta dirección) y por la asamblea de accionistas (OCDE, 2005). Este sistema de organización se asemeja al empleado en el campo del sector público como es el caso del ámbito del sector educativo o el sector salud.

La investigación aborda un análisis bibliométrico, este se caracteriza por la utilización de medios matemáticos y estadísticos para el análisis de un área del conocimiento que puede determinar la situación y el progreso de una disciplina (Durisin, Calabretta $\&$ Parmeggiani, 2010). Son vistos como una fuente que ayuda a identificar los objetivos y alcances de las diversas disciplinas (Lee \& Vicente, 2005). Por lo tanto, existen diversas técnicas para emprender un estudio bibliométrico, estas se pueden agrupar principalmente por los indicadores cuantitativos que se caracterizan por establecer el número de publicaciones o por los indicadores de impacto, este último se basa en el número de citas que se encuentran en los trabajos (Ramos-Rodríguez \& Ruiz-Navarro, 2004; Bordons \& Zulueta, 1999). 
Los estudios bibliométricos realizados en las revistas académicas del Intitute for Scientific Information (ISI) son valoradas como un indicador de calidad (Legge \& Devore, 1987), esta fuente de información posee un alto prestigio debido a su gran impacto, a su evaluación y a su difusión (Kellough \& Pitts, 2005).

Existe en la literatura estudios bibliométricos previos sobre el GC como es el realizado por Durisin \& Puzone (2009), su investigación se ha caracterizado principalmente por analizar la evolución y la maduración del GC en el ámbito privado hasta el punto de situar al GC como una disciplina. Además, se ha encontrado una investigación reciente en el campo objeto de estudio como es la realizada por Flórez, López \& López (2014b) esta aborda un estudio bibliométrico sobre el GC en el sector público, su análisis identifica el traslado del concepto del GC al ámbito público y su implementación en algunos campos del sector público.

El objetivo de la presente investigación se centra en realizar una revisión de la literatura en las revistas ISI del área del conocimiento de la Administración Pública, se pretende analizar el estado actual, los avances, las temáticas, los países más influyentes y las universidades que investigan en materia de GC en el sector público, el periodo seleccionado comprende los años 2007-2015. La inclinación por este periodo de tiempo responde a los escándalos financieros ocurridos en este periodo que ha desencadenado una política de austeridad, especialmente en el sector público, ocasionando nuevos planteamientos de organización.

Por lo tanto, el trabajo se estructura de la siguiente forma, en la siguiente sección abordaremos el GC en el sector público, en la sección tercera proporcionaremos la metodología empleada en la investigación, seguidamente estableceremos los análisis de los resultados y por último finalizaremos con las principales conclusiones.

\section{El GC en el sector público}

En las últimas décadas el sector público ha venido buscando nuevos sistemas de organización que le garantice ser más eficiente y eficaz en la gestión, esencialmente los esfuerzos de la Administración
Pública se han centrado en el ánimo de reducir la burocracia y el déficit público (Dent, Gestel \& Teelken, 2007). Así, a finales de la década de los 70 e inicio de los 80 la New Public Management -nueva gestión pública- irrumpe en el contexto del sector público (Hacen, 2011). A partir de los años 90, la nueva gestión pública toma una mayor influencia a nivel mundial, su definición es caracterizada por la prestación de un buen servicio a los ciudadanos donde la responsabilidad y las buenas prácticas son los pilares esenciales para mejorar las organizaciones del sector público (Hood, 1991). En la actualidad, la búsqueda de una mejor gestión en el ámbito público es conocida como el GC.

Autores como Cadbury (1992) han contribuido a introducir el concepto del GC en el campo del sector público, su aportación se centró en tomar algunos componentes del GC empleados en el sector privado y trasladarlos a la Administración Pública con el ánimo de construir un modelo de gestión más eficiente y eficaz, este aporte culminó con el establecimiento de una serie de aspectos orientados al desarrollo de códigos de buenas prácticas.

Los planteamientos del GC en el sector público consisten en un modelo de gobernanza que establece un sistema de organización donde básicamente la gestión debe ser más transparente y con una mayor rendición de cuentas (Rhodes, 1996). Por otra parte, los aportes hechos por el GC han ayudado a incorporar un nuevo estilo de gestión en la Administración Pública. Su concepto ha contribuido a reorganizar y modernizar las estructuras de dirección de algunos campos del sector público, esta empieza a basarse en el camino hacia la eficiencia, en la transformación de los procedimientos, en la cultura organizativa y en la organización de sus órganos de dirección (Ferlie, Ashburner \& Fitzgerald, 1995).

Los países que iniciaron la implementación del GC en el campo del sector público fueron el Reino Unido en la administración de Margaret Thatcher y en los Estados Unidos en la presencia de Ronald Reagan, que en los años 80 promovieron la modernización de la Administración Pública, los cambios se centraron principalmente en sectores como la educación y la sanidad (Lazonick \& O'Sullivan, 


\section{EL GOBIERNO CORPORATIVO EN EL ÁMBITO DEL SECTOR PÚBLICO: UN ESTUDIO BIBLIOMÉTRICO EN LAS REVISTAS UBICADAS EN EL ÁREA DE ADMINISTRACIÓN PÚBLICA}

2000; Taylor, 2013). Por otra parte, países como Australia, Nueva Zelanda e incluso Canadá iniciaron la implementación del GC en el sector público en estas mismas áreas.

Las principales contribuciones realizadas sobre el GC en el sector público se han realizado en áreas como la sanidad (Clatworthy, Mellett \& Peel, 2000); la mejora de los gobiernos locales (Whiteoak, 1996); las estructuras de dirección y gobierno en las universidades (Carnegie \& Tuck, 2010). Otros estudios sobre el GC en el ámbito público han girado en la construcción de principios, normas, informes o códigos de buenas prácticas (Cadbury, 1992; Ryan \& Ng, 2000). De hecho, esta última temática ha tenido una repercusión a nivel mundial ya que la Organización para la Cooperación y el Desarrollo Económico OCDE (2004) emitió los principios del GC para las organizaciones públicas y privadas con un carácter voluntario.

La estructura del GC en el campo del sector público se ha caracterizado generalmente por la incorporación de procesos más gerenciales en la dirección de las organizaciones y por reducir el número de consejos en los órganos de dirección. Un ejemplo de estos cambios son los producidos en el ámbito de la educación superior en especial los realizados en las universidades de la cultura anglosajona (Rodgers, Freeman, Williams \& Kane, 2011). La implementación del GC en el ámbito educativo ha generado que las universidades de la cultura anglosajona se sitúen en su mayoría en los primeros puestos del top 100 (Academic Ranking of World Universities, 2015)

Las aportaciones sobre el GC en el ámbito público son escasas respecto a las realizadas por el sector privado (Flórez, et al. 2014a, 2014b). A pesar de estas limitaciones, la implementación del GC se ha empezado a extender en el sector público, principalmente debido a las reformas administrativas impulsadas por numerosos países como el Reino Unido, Australia o Canadá (Hodges, Wright \& Keasey, 1996; Bozec, Zéghal \& Boujenoui, 2004; Howard \& Seth-Purdie, 2005; Ryan, Stanley \& Nelson, 2002). De hecho, en la medida que los países de la cultura anglosajona promuevan la aplicación del GC en el sector público indica en gran medida un proceso de convergencia y armonización de los demás países hacia este modelo (Cernat, 2004; James, 2001).

\section{Investigación empírica}

\subsection{Selección de la muestra}

Para realizar el análisis de la temática del GC en el sector público, como primera medida se ha optado por tomar en nuestra investigación las revistas de mayor prestigio e impacto internacional establecidas en el Journal Citation Reports (JCR) correspondiente al año 2015, último año publicado por JCR. Específicamente analizaremos el área de Public Administration con un total de 47 revistas (tabla 1 ).

La inclinación por este medio se debe en gran medida a que las revistas académicas poseen gran prestigio internacional (Braadbaart \& Yusnandarshah, 2008); son vistas como indicadores de alta calidad académica (Legge \& Devore, 1987); son utilizadas por los diversos investigadores para difundir nuevos conocimientos (Nord \& Nord, 1995) y, a su vez, las revistas indexadas generan mayor valor añadido (Garand, 1990).

Como el campo del conocimiento a estudiar sobre el GC es muy amplio, como criterio, se ha procedido a tomar un periodo de tiempo que será objeto de estudio. La elección de dicho periodo comprende los años 2007-2015. La decisión de tomar este periodo responde a los escándalos financieros y de corrupciones ocurridas a principios del siglo XXI que ha generado una mayor intervención y control por parte del Estado, así el interés por profundizar y establecer alternativas, ha llevado a tratar esta temática con mayor interés.

Una vez establecido el campo de estudio y seleccionadas las 47 revistas académicas del área de Public Administration con las que trabajaremos. Se procedió en primer lugar, a clasificar la muestra objeto de estudio de acuerdo a su factor de impacto, este debe ser superior a 0,25 o con más de 50 citas (Plüper \& Radaelli, 2004). De esta forma se ha obtenido una muestra final de 43 revistas académicas que suponen un 91,49 (tabla 1). 
Tabla 1. Contribuciones del GC en las revistas académicas del área temática Public Administration, periodo 2007-2015

\begin{tabular}{|c|c|c|c|c|c|c|c|c|}
\hline Ranking & Nombre de las revistas & \begin{tabular}{|c|}
$\begin{array}{c}\text { Abreviación } \\
\text { de las } \\
\text { revibtas }\end{array}$ \\
\end{tabular} & \begin{tabular}{|c|} 
Journal \\
Impact \\
Factor 2015 \\
\end{tabular} & $\begin{array}{l}\text { Total } \\
\text { Cites } \\
\end{array}$ & ISSN & \begin{tabular}{|c|} 
Artículos \\
encontrados sobre \\
GC ámbito público.
\end{tabular} & \begin{tabular}{|c|} 
Artículos \\
encontrados sobre \\
GC ámbito privado.
\end{tabular} & Total de Articulos \\
\hline 1 & JOURNALOF PUBLIC ADMINISTRATION RESEARCH AND THEORY & JPART & 3.893 & 3,121 & 1053-1858 & 2 & $\cdot$ & 362 \\
\hline 2 & GOVERNANCE-AN INIERNATIONAL JOURNAL OF POLICY ADMINISTRATION AND INSIIIUIIONS & $\mathrm{G}$ & 3.424 & 1,204 & 0952-1895 & 1 & $=$ & 243 \\
\hline 3 & REGULATION \& GOVERNANCE & RG & 2.724 & 480 & $1748-5983$ & - & 3 & 225 \\
\hline 4 & PUBLIC ADMINISTRATION REVIEW & PAR & 2.636 & 5,183 & 0033-3352 & 2 & - & 705 \\
\hline 5 & JOURNAL OF POLICY ANALYSIS AND MANAGEMENT & JPAM & 2.329 & 1,731 & $0276-8739$ & - & - & 322 \\
\hline 6 & CLIMATE POLCY & $\mathrm{CP}$ & 1.980 & 1,117 & 1469-3062 & $\cdot$ & $\cdot$ & 398 \\
\hline 7 & JOURNAL OF EUROPEAN PUBLC POLCY & JEPP & 1.964 & 2,308 & $1350-1763$ & - & 1 & 668 \\
\hline 8 & PUBLC ADMINISTRATION & $\mathrm{PA}$ & 1.922 & 2,257 & $0033-3298$ & 16 & - & 542 \\
\hline 9 & PUBLIC MANAGEMENT REVIEW & PMR & 1.872 & 1,184 & 1471-9037 & 5 & . & 446 \\
\hline 10 & POLICY STUDIES JOURNAL & PSJ & 1.765 & 1,178 & 0190-292X & 1 & - & 294 \\
\hline 11 & ENVIRONMENT AND PLANNING C-GOVERNMENT AND POLICY & EPGP & 1.664 & 1,443 & $0263-774 \mathrm{X}$ & 2 & - & 568 \\
\hline 12 & POLICY SCIENCES & PS & 1.641 & 1,182 & $0032-2687$ & . & - & 165 \\
\hline 13 & JOURNAL OF EUROPEAN SOCIAL POLICY & JESP & 1.426 & 950 & $0958-9287$ & 1 & - & 252 \\
\hline 14 & JOURNALOF ACCOUNIING AND PUBLIC POLICY & JAPP & 1.317 & 1,041 & $0278-4254$ & 2 & 33 & 270 \\
\hline 15 & AMERICAN REVIEW OF PUBLLC ADMINISTRATION & ARPA & 1.260 & 859 & $0275-0740$ & 2 & - & 301 \\
\hline 16 & INIERNATIONAL PUBLLC MANAGEMENT JOURNAL & IPMJ & 1.233 & 531 & 1096-7494 & - & - & 181 \\
\hline 16 & SCIENCE AND PUBLC POLICY & SPP & 1.233 & 1,104 & $0302-3427$ & - & $\cdot$ & 542 \\
\hline 18 & REVIEW OF PUBLIC PERSONNEL ADMINISTRATION & RPPA & 1.222 & 426 & 0734-371X & - & - & 183 \\
\hline 19 & POLICY AND POUTICS & $\mathrm{PP}$ & 1.200 & 537 & $0305-5736$ & - & - & 294 \\
\hline 20 & REVIEW OF POLICY RESEARCH & RPR & 1.167 & 504 & 1541-132X & - & 1 & 262 \\
\hline 21 & JOURNAL OF SOCIALPOLUCY & JSP & 1.151 & 844 & $0047-2794$ & - & - & 313 \\
\hline 22 & SOCIAL POLICY \& ADMINISTRATION & SPA & 1.069 & 807 & $0144-5596$ & 1 & $\cdot$ & 389 \\
\hline 23 & JOURNALOF PUBLIC POLICY & JPP & 1.000 & 605 & $0143-814 \mathrm{X}$ & 1 & - & 136 \\
\hline 24 & POLCY AND SOCETY & PS & 0.944 & 278 & $1449-4035$ & 2 & - & 245 \\
\hline 25 & PUBLC PERFORMANCE \& MANAGEMENT REVIEW & PPMR & 0.909 & 414 & $1530-9576$ & 1 & - & 244 \\
\hline 26 & ADMINISTRATION \& SOCIETY & AS & 0.891 & 912 & 0095-3997 & 4 & - & 352 \\
\hline 27 & POLICY STUDIES & PS & 0.873 & 327 & $0144-2872$ & 3 & 2 & 287 \\
\hline 28 & PUBLC ADMINISTRATION AND DEVELOPMENT & PAD & 0.821 & 639 & 0271-2075 & 2 & - & 269 \\
\hline 29 & LOCALGOVERNMENT STUDIES & LGS & 0.804 & 449 & 0300-3930 & - & - & 356 \\
\hline 30 & LEXLOCALLS-JOURNAL OF LOCAL SEIF-GOVERNMENT & LISG & 0.802 & 117 & $1581-5374$ & $\cdot$ & - & 227 \\
\hline 31 & ADMINISTRATION IN SOCIAL WORK & ASW & 0.750 & 376 & $0364-3107$ & 1 & - & 248 \\
\hline 32 & PUBLC MONEY \& MANAGEMENT & PMM & 0.718 & 656 & $0954-0962$ & 7 & - & 438 \\
\hline 33 & INIERNATIONAL REVIEW OF ADMINISTRATIVE SCIENCES & $\mathbb{R A S}$ & 0.716 & 591 & $0020-8523$ & 8 & - & 321 \\
\hline 34 & AUSTRALLAN JOURNAL OF PUBLIC ADMINISTRATION & APA & 0.667 & 631 & 0313-6647 & 3 & $=$ & 312 \\
\hline 35 & NONPROFIT MANAGEMENT \& LEADERSHIP & NML & 0.653 & 620 & 1048-6682 & 1 & 9 & 222 \\
\hline 36 & JOURNAL OF COMPARATIVE POLICY ANALYSIS & JCPA & 0.642 & 323 & $1387-6988$ & - & - & 224 \\
\hline 37 & CONIEMPORARY ECONOMIC POLICY & CEP & 0.602 & 672 & $1074-3529$ & - & - & 414 \\
\hline 38 & PUBLLC PERSONNEL MANAGEMENT & PPM & 0.596 & 398 & $0091-0260$ & 1 & - & 250 \\
\hline 39 & CANADIAN PUBULC POLICY-ANALYSE DE POLITQUES & CPPAP & 0.475 & 413 & $0317-0861$ & - & - & 307 \\
\hline 40 & JOURNAL OF HOMELAND SECURITY AND EMERGENCY MANAGEMENT & JHSEM & 0.466 & 266 & $2194-6361$ & - & - & 310 \\
\hline 41 & HUMAN SERVICE ORGANIZATIONS MANAGEMENT LEADERSHIP \& GOVERNANCE & HSOMLG & 0.429 & 18 & $2330-3131$ & - & - & 248 \\
\hline 42 & CANADIAN PUBLUC ADMINISTRATION-ADMINISTRATION PUBLLQUE DU CANADA & CPAAPC & 0.300 & 201 & $0008-4840$ & 4 & 1 & 232 \\
\hline \multirow[t]{4}{*}{43} & TRANSYLVANIAN REVIEW OF ADMINISTRATIVE SCIENCES & TRAS & 0.271 & 87 & $1842-2845$ & - & - & 395 \\
\hline & \multicolumn{2}{|l|}{ Total revistas } & \multicolumn{2}{|l|}{43} & $91,49 \%$ & & & \\
\hline & \multicolumn{5}{|l|}{ Total articulos } & 73 & 50 & 13.962 \\
\hline & \multicolumn{5}{|l|}{ Porcentaje total artículos sobre GC } & $0,52 \%$ & $0,36 \%$ & \\
\hline 44 & CIVIL SZEMLE & CS & 0.136 & 21 & $1786-3341$ & & & \\
\hline 45 & REVISTA DEL CLAD REFORMA Y DEMOCRACIA & RCLADRD & 0.111 & 27 & 1315-2378 & & & \\
\hline 46 & GESTIÓN Y POLIICA PÚBLCA & GPP & 0.098 & 31 & $1405-1079$ & & & \\
\hline 47 & AMME IDARESI DERGISI & AID & 0.019 & 17 & $\mid 1300-1795$ & & & \\
\hline
\end{tabular}

Fuente: Elaboración propia. 
En segundo lugar se procedió a la selección de los artículos, para la revisión, en primer lugar se realizó una búsqueda con los siguientes parámetros: se analizó el título y seguidamente el resumen (Lan \& Anders, 2000; Plümper \& Radaelli, 2004), a continuación se revisó las palabras claves (Bartki, Rivard \& Talbot, 1988); por último, si no fueron suficientes estos criterios, se realizó la lectura total del artículo. Fruto de esta tediosa labor, se estableció una base de datos compuesta de 13.962 artículos publicados en las 43 revistas ISI, de los cuales, 27 revistas publican aspectos referentes al GC tanto del sector público como del privado obteniendo 123 artículos. De esta forma, trabajaremos con 24 revistas que nos han proporcionado 73 artículos del ámbito público (Ilustración 1).

\subsection{Metodología}

Con el fin de analizar la temática del GC en el sector público y para alcanzar los objetivos propuestos, cada uno de los artículos encontrados sobre GC fue catalogado de acuerdo a su año de publicación, el nombre de la revista, la filiación institucional (universidad) de los autores, el país de la revista que publica la investigación, el idioma en el que han sido publicados los trabajos, las principales temáticas tratadas y las principales metodologías utilizadas. En el caso de que algún artículo seleccionado en la muestra objeto de estudio obtenga más de una metodología y para evitar posibles errores se ha procedido a tomar la metodología principal de la investigación llevada a cabo.

En el análisis de contenido de los artículos encontrados sobre GC en las revistas ISI en el área temática de Public Administration, se utilizó la base de datos de la biblioteca de la Universidad de Granada-España, donde se empleó las colecciones de revistas de ABI/INFORM, Wiley-Blackwell 2010 Full Collection, Oxford Journals, Taylor \& Francis Online, Business Source Complete, SAGE Premier 2012, Taylor \& Francis Library (SSH and ST) y Cambridge Journals Online. Para llevar a cabo la búsqueda, se utilizó palabras claves como "Corporate Governance" "Board Directors" "Code" "Good Governance" "Public Sector".

Para obtener los datos necesarios a fin de realizar una revisión exhaustiva y poder recabar la informa-

Ilustración 1. Artículos sobre el GC en el sector público en las revistas ISI (2007-2015)

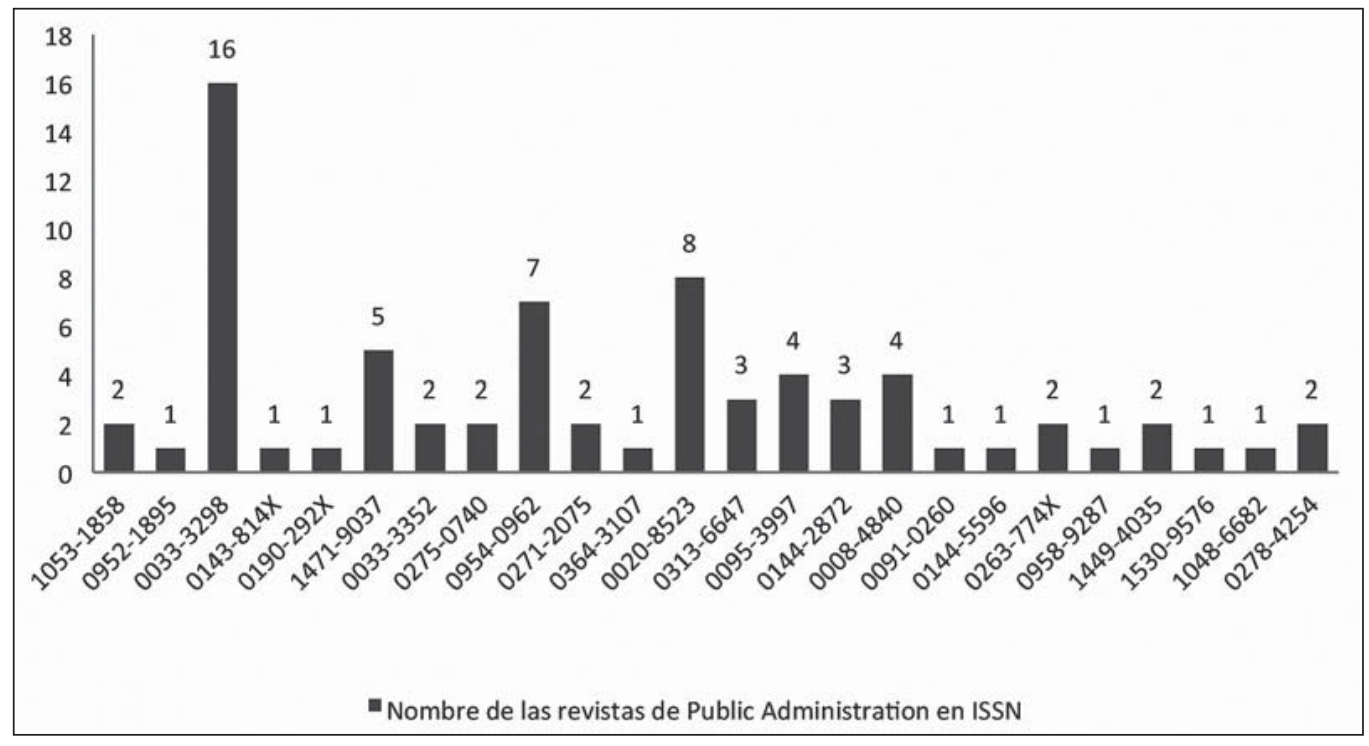

Fuente: Elaboración propia. 
ción necesaria para la presente investigación, esta se realizó entre los meses de junio y agosto del año 2016.

\section{Análisis de los resultados}

De acuerdo a los datos obtenidos en la llustración 2, el GC en el sector público en los últimos años tiene cada vez más presencia en las revistas del ámbito público, su irrupción en esta área del conocimiento se debe esencialmente a la fuerte influencia generada por la cultura anglosajona y al interés que genera en la comunidad académica los aspectos como la transparencia, la rendición de cuentas, la eficiencia y la eficacia en entidades del sector público.

De las 47 publicaciones catalogadas en la sección de Public Administration 24 revistas son las que abordan la temática relacionada con el GC en el campo del sector público. Según el análisis bibliométrico desarrollado, se ha podido identificar 73 artículos de los cuales más de un 60 se encuentran publicados en las siguientes revistas: Public Administration $(21,92)$, International Review of Administrative Sciences $(10,96)$, Public Money \& Management (9,59), Public Management Review $(6,85)$; Canadian Public Administration-Administration Publique DU Canadá $(5,48)$ y Administration \& Society $(5,48)$.

En las investigaciones analizadas sobre el GC en el sector público y tal como se han señalado en la tabla 2 , las principales temáticas tratadas según su orden de importancia son los siguientes estudios: i) estilos de gobierno y estructuras de organización $(23,29)$; ii) reforma y mejoras en la administración pública $(16,44)$; iii) transparencia, responsabilidad, rendición de cuentas e internet $(12,33)$ y privatización de las empresas públicas y austeridad $(12,33)$; iv) análisis teórico del GC $(8,22)$; y v) código de buenas prácticas y buen gobierno $(6,85)$, eficiencias en las organización del sector público $(6,85)$, buen gobierno y gobernanza $(6,85)$, y gobierno central y descentralización de los gobiernos locales $(6,85)$.

Ilsutración 2. Distribución temporal de los artículos encontrados sobre el GC en el catálogo de Public Administration, periodo 2007-2015

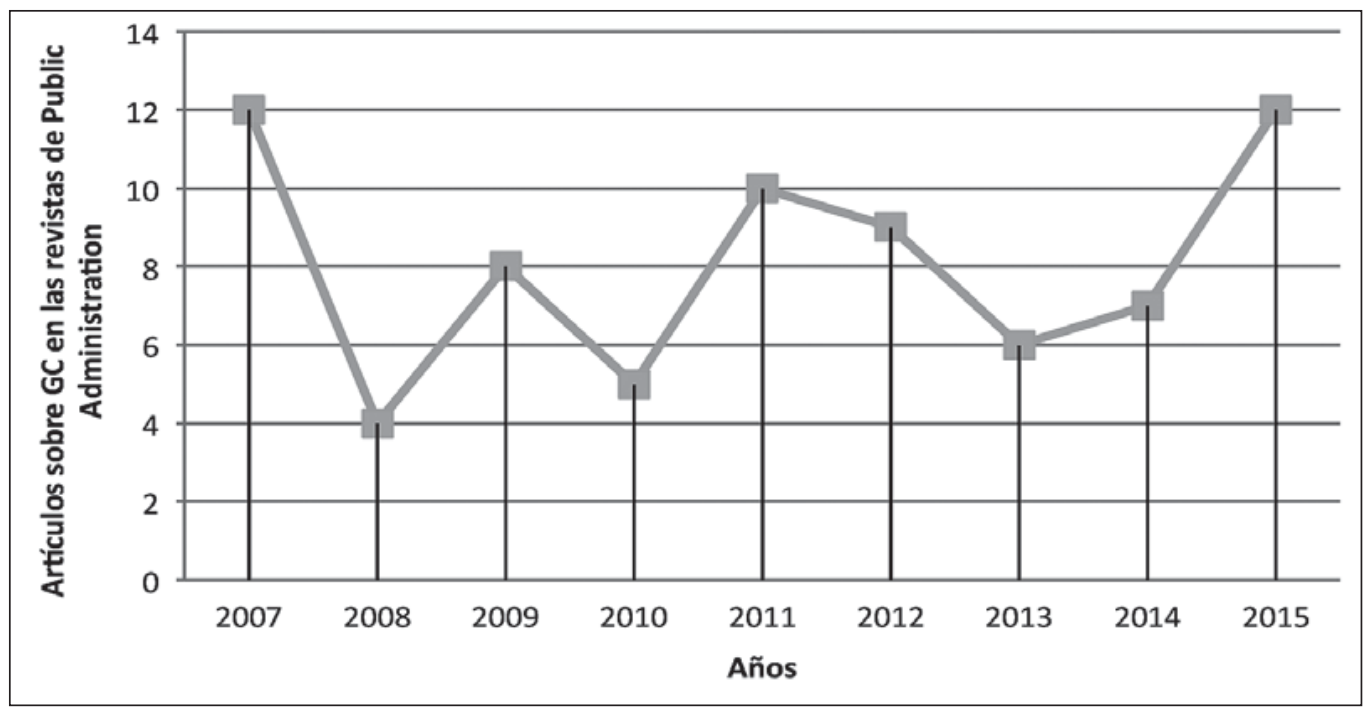

Fuente: Elaboración propia. 


\section{EL GOBIERNO CORPORATIVO EN EL ÁMBITO DEL SECTOR PÚBLICO: UN ESTUDIO BIBLIOMÉTRICO EN LAS REVISTAS UBICADAS EN EL ÁREA DE ADMINISTRACIÓN PÚBLICA}

Tabla 2. Principales temáticas analizadas por las revistas de Public Administration sobre el GC en el sector público

\begin{tabular}{|c|c|c|c|c|c|c|c|c|c|c|c|}
\hline Temáticas & $\begin{array}{c}\text { Reformas y } \\
\text { mejoras en la } \\
\text { Administración } \\
\text { y el Servicio } \\
\text { Público }\end{array}$ & $\begin{array}{l}\text { Estilos de } \\
\text { gobierno y } \\
\text { estructuras } \\
\text { de organi- } \\
\text { zación }\end{array}$ & $\begin{array}{l}\text { Transparen- } \\
\text { cia, respon- } \\
\text { sabilidad, } \\
\text { rendición } \\
\text { de cuentas } \\
\text { e internet }\end{array}$ & $\begin{array}{l}\text { Códi- } \\
\text { gos de } \\
\text { buenas } \\
\text { prácticas } \\
\text { y buen } \\
\text { gobierno }\end{array}$ & $\begin{array}{c}\text { Privati- } \\
\text { zación } \\
\text { de las } \\
\text { empresas } \\
\text { públicas y } \\
\text { austeridad }\end{array}$ & $\begin{array}{l}\text { Eficiencia } \\
\text { en las } \\
\text { organi- } \\
\text { zaciones } \\
\text { del sector } \\
\text { público }\end{array}$ & $\begin{array}{c}\text { Análisis } \\
\text { teórico } \\
\text { del GC }\end{array}$ & $\begin{array}{c}\text { Buen } \\
\text { gobierno y } \\
\text { gobernanza }\end{array}$ & $\begin{array}{l}\text { Gobierno } \\
\text { central y la } \\
\text { descentrali- } \\
\text { zación de los } \\
\text { gobiernos } \\
\text { locales }\end{array}$ & Total & $\%$ \\
\hline JPART & 1 & & & & & & 1 & & & 2 & 2,74 \\
\hline G & 1 & & & & & & & & & 1 & 1,37 \\
\hline PA & 3 & 5 & 1 & 1 & & 1 & 2 & 2 & 1 & 16 & 21,92 \\
\hline JPP & & & & & 1 & & & & & 1 & 1,37 \\
\hline PSJ & & 1 & & & & & & & & 1 & 1,37 \\
\hline PMR & & 3 & & & & & 1 & 1 & & 5 & 6,85 \\
\hline PAR & 1 & & & & 1 & & & & & 2 & 2,74 \\
\hline ARPA & & & & & & 1 & 1 & & & 2 & 2,74 \\
\hline PMM & 1 & 1 & 1 & & 1 & 2 & & 1 & & 7 & 9,59 \\
\hline PAD & & & & 1 & & & & & 1 & 2 & 2,74 \\
\hline ASW & & & & 1 & & & & & & 1 & 1,37 \\
\hline IRAS & & 1 & 3 & 1 & & & & & 3 & 8 & 10,96 \\
\hline AJPA & & & 1 & 1 & & & & 1 & & 3 & 4,11 \\
\hline AS & 2 & 1 & & & & & 1 & & & 4 & 5,48 \\
\hline PS & & & 1 & & 1 & 1 & & & & 3 & 4,11 \\
\hline CPAAPC & 2 & & 1 & & 1 & & & & & 4 & 5,48 \\
\hline EPGP & & & 1 & & 1 & & & & & 2 & 2,74 \\
\hline JESP & & & & & 1 & & & & & 1 & 1,37 \\
\hline JAPP & & 1 & & & 1 & & & & & 2 & 2,74 \\
\hline SPA & 1 & & & & & & & & & 1 & 1,37 \\
\hline PS & & 2 & & & & & & & & 2 & 2,74 \\
\hline PPMR & & & & & 1 & & & & & 1 & 1,37 \\
\hline NML & & 1 & & & & & & & & 1 & 1,37 \\
\hline PPM & & 1 & & & & & & & & 1 & 1,37 \\
\hline Total & 12 & 17 & 9 & 5 & 9 & 5 & 6 & 5 & 5 & 73 & \\
\hline$\%$ & 16,44 & 23,29 & 12,33 & 6,85 & 12,33 & 6,85 & 8,22 & 6,85 & 6,85 & & \\
\hline
\end{tabular}

Fuente: Elaboración propia. 
Las tendencias en las temáticas obtenidas sobre el GC en el sector público se han implementado en sectores como la educación superior así como lo expone De Boer, Enders \& Leisyte, (2007), su trabajo aborda una de las temáticas más frecuentes como es reformas en la Administración Pública dirigidas a los sistemas de organización en las universidades específicamente a los cambios efectuados en la autonomía universitaria. Otros autores como Carnegie \& Tuck (2010) ponen el acento en su investigación en los valores comerciales que poseen las universidades y para ellos establecen tres factores claves como son la gobernanza académica, la gestión de la empresa y el GC.

Por otra parte, existen otros trabajos como el de Stewart et al. (2012), que aborda la temática del GC sobre la transparencia, la responsabilidad, la rendición de cuentas e internet en sectores como los departamentos del Gobierno australiano donde analizan el alcance de los informes de gestión divulgados en las páginas webs. No obstante, existen trabajos del GC en sectores como la sanidad pública del Reino Unido, su temática ha girado sobre los estilos de gobierno y las estructuras de organización de los consejos de administración en los centros de salud británicos (Veronesi \& Keasey, 2010).

En cuanto a las metodologías usadas en los artículos, hemos podido identificar una fuerte inclinación al uso de los métodos de investigación empíricos, con un 83,56 , sobre los no empíricos 16,44 . Entre los métodos empíricos usados debemos mencionar que la herramienta cualitativa es la más utilizada $(72,60)$ superando a las herramientas cuantitativas $(10,96)$ (tabla 3). De hecho, la metodología cualitativa más empleada es la de los estudios de casos $(30,14)$ mientras que en los estudios cuantitativos la herramienta más utilizada es el análisis de regresión $(37,50)$.

Tabla 3. Metodologías empleadas para analizar cada una de las temáticas relacionadas con el GC

\begin{tabular}{|c|c|c|c|c|c|c|c|c|c|c|c|}
\hline \multirow{3}{*}{ Temáticas/Metodologías } & \multirow{3}{*}{$\begin{array}{c}\text { E. No } \\
\text { Empíricos } \\
16,44\end{array}$} & \multicolumn{10}{|c|}{ Métodos Empíricos 83,56 } \\
\hline & & \multicolumn{5}{|c|}{$\begin{array}{c}\text { Metodologías Cualitativas } \\
72,60 \\
\end{array}$} & \multicolumn{4}{|c|}{$\begin{array}{c}\text { Metodologías Cuantitativas } \\
10,96 \\
\end{array}$} & \multirow[b]{2}{*}{ Total } \\
\hline & & ANCOP & AC & EC & MC & AD & ANCOP & AC & AREG & ED & \\
\hline Reformas y mejoras en la Administración Pública. & 3 & & 3 & 4 & 1 & & 1 & & 1 & & 13 \\
\hline Estilos de gobierno y estructuras de organización. & 4 & 1 & & 5 & 2 & 1 & & 1 & 1 & 1 & 16 \\
\hline $\begin{array}{l}\text { Transparencia, responsabilidad, rendición de cuentas } \\
\text { e internet. }\end{array}$ & & 2 & & 3 & 1 & 2 & 1 & & & & 9 \\
\hline Códigos de buenas prácticas y buen gobierno. & 1 & & 1 & & 1 & 2 & & & & & 5 \\
\hline Privatización de las empresas públicas y austeridad. & 2 & 3 & & 2 & & 1 & & & 1 & & 9 \\
\hline Eficiencia en las organizaciones del sector público. & 1 & & 1 & 1 & 2 & & & & & & 5 \\
\hline Análisis teórico del GC. & & 2 & 2 & & 1 & & & 1 & & & 6 \\
\hline Buen gobierno y gobernanza. & 1 & & & 3 & & 1 & & & & & 5 \\
\hline $\begin{array}{l}\text { Gobierno central y la descentralización de los gobiernos } \\
\text { locales. }\end{array}$ & & & & 4 & & 1 & & & & & 5 \\
\hline Total & 12 & 8 & 7 & 22 & 8 & 8 & 2 & 2 & 3 & 1 & \\
\hline
\end{tabular}

Nota: Lista de abreviaturas: ANCOP: Análisis Comparativo, AC: Análisis de Contenido, EC: Estudios de Casos, NOE: No Empíricos, AD: Análisis Descriptivos, MC: Multi Casos, ED: Estadística Descriptiva, AREG: Análisis de Regresión.

Fuente: Elaboración propia. 
Respecto a los principales países que realizan una mayor aportación en las investigaciones sobre el GC en el campo del sector púbico estos son: i) Reino Unido 68,49; ii) Estados Unidos 27,40; y iii) Australia con un 4,11 (Ilustración 3. Entre las universidades ubicadas en el Reino Unido que mayores aportaciones realizan sobre el GC encontramos a las universidades públicas como la de Birmingham, Nottingham, Escuela Universitaria de Londres (UCL), Plymouth e incluso la universidad de Oxford que enfocan sus investigaciones a temáticas como el análisis teórico del GC, la eficiencia de las organizaciones del sector público, la privatización de las empresas públicas y la transparencia, la responsabilidad y la rendición de cuentas (tabla 2).

En cuanto a las investigaciones del GC en el sector público procedentes de Norte América $(19,18)$ - el 10,96 pertenecen a universidades de los Estados Unidos como la de Stanford, Massachusetts, Maryland, Pennsylvania, Carolina del Norte A \& T, Dayton y Ohio y el 8,22 son de autores canadienses, las investigaciones provienen de universidades pú- blicas tales como la de Quebec, Ottawa, Dalhousie, Saint Mary's y Carleton (Tabla 4).

En relación a otros resultados importantes se encuentran las universidades australianas (9,59). Las investigaciones provienen de autores que se encuentran adscritos a universidades como Tasmania, Melbourne, Sídney, Queensland, Ballarat y Griffith sus trabajos se enmarcan sobre el buen gobierno y gobernanza, código de buenas prácticas e incluso la transparencia, la responsabilidad y la rendición de cuentas.

Los trabajos no solo provienen del Reino Unido, Norte América o Australia. Una parte de las investigaciones proceden de países como los Países Bajos $(6,85)$ y las universidades que contribuyen a su análisis son la de Twente, Ámsterdam y Utrecht. Otras universidades como la de Bremen y Konstanz en Alemania, las universidades de Chongqing, Xiamen y Hong Kong en China, las universidades de Roma y Siena en Italia y las universidades de Zurich o Geneve en Suiza son la que tratan más la temática del GC en el sector público con un $(4,11$, respectivamente).

Ilustración 3. Revistas por países que publican artículos del GC en el sector público

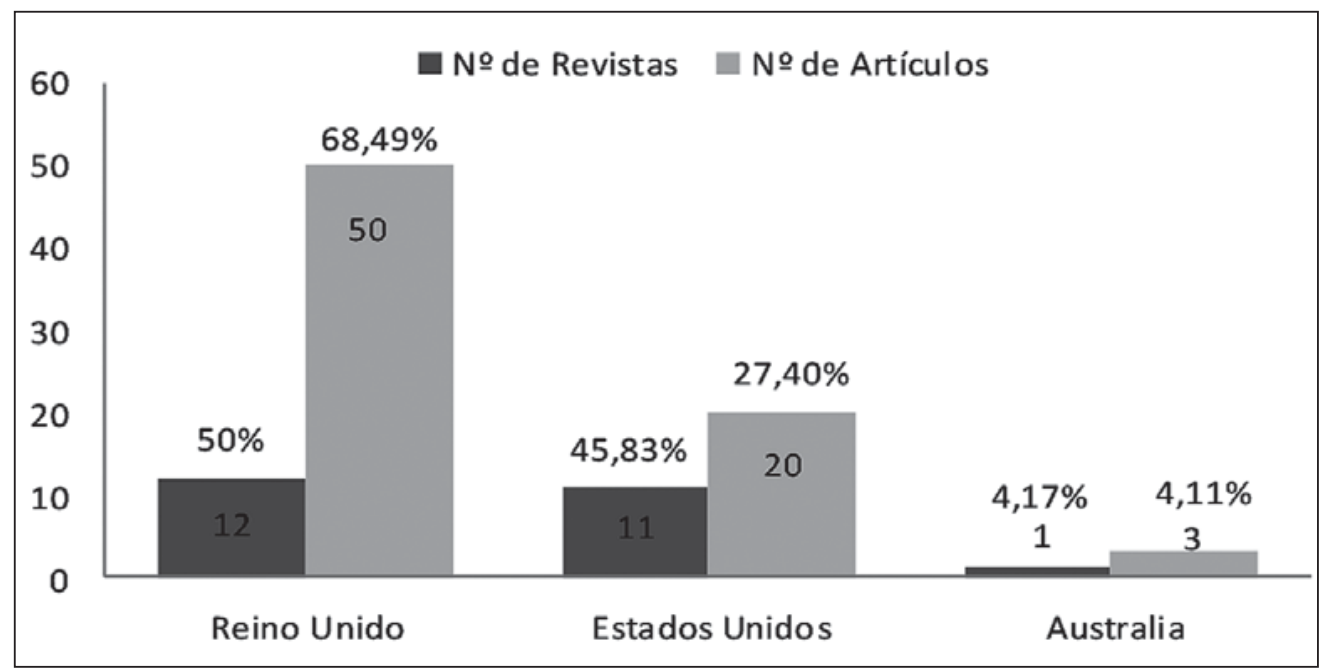

Fuente: Elaboración propia. 
Tabla 4. Temas de investigación analizados por cada uno de los países y sus universidades

\begin{tabular}{|c|c|c|c|c|c|c|c|c|c|c|c|c|c|c|c|c|c|c|c|}
\hline Temáticas & $\begin{array}{l}\text { 을 } \\
\text { 을 } \\
\text { 읋 }\end{array}$ & 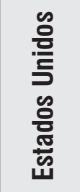 & 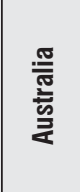 & 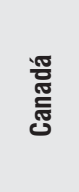 & 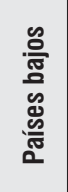 & 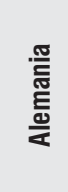 & 䄈 & 袈 & $\stackrel{\stackrel{N}{N}}{\bar{B}}$ & 总 & $\frac{0}{5}$ & 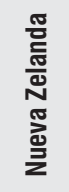 & 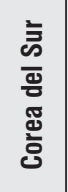 & 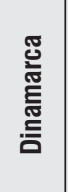 & 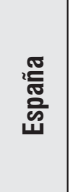 & $\begin{array}{l}\text { 訔 } \\
\text { 总 }\end{array}$ & 言 & 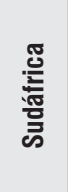 & 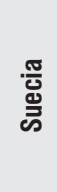 \\
\hline $\begin{array}{l}\text { Reformas y } \\
\text { mejoras en la } \\
\text { Administra- } \\
\text { ción Pública. }\end{array}$ & 13,04 & 25,00 & 28,57 & 33,33 & 20 & & & & & & 50 & 100 & & & 100 & & & & \\
\hline $\begin{array}{l}\text { Estilos de } \\
\text { gobierno y } \\
\text { estructuras de } \\
\text { organización. }\end{array}$ & 17,39 & 37,50 & & 16,67 & 40 & 33,33 & 66,67 & 66,67 & & & & & & & & & & & 100 \\
\hline $\begin{array}{l}\text { Transparencia, } \\
\text { responsabili- } \\
\text { dad, rendición } \\
\text { de cuentas e } \\
\text { internet. }\end{array}$ & 17,39 & & 28,57 & 16,67 & & 33,33 & & & 33,33 & & & & & & & & & & \\
\hline $\begin{array}{l}\text { Códigos de } \\
\text { buenas prác- } \\
\text { ticas y buen } \\
\text { gobierno. }\end{array}$ & 4,35 & 25,00 & 14,29 & & & & & & 33,33 & & & & & & & & & & \\
\hline $\begin{array}{l}\text { Privatización } \\
\text { de las empre- } \\
\text { sas públicas } \\
\text { y austeridad. }\end{array}$ & 13,04 & & & 16,67 & & 33,33 & 33,33 & & & 50 & & & & 100 & & & 100 & & \\
\hline $\begin{array}{l}\text { Eficiencia en } \\
\text { las organiza- } \\
\text { ciones del } \\
\text { sector público. }\end{array}$ & 9,09 & & & & & & & 33,33 & & & 50 & & 100 & & & & & & \\
\hline $\begin{array}{l}\text { Análisis } \\
\text { teórico del GC. }\end{array}$ & 13,64 & & 14,29 & & 20 & & & & 33,33 & & & & & & & & & & \\
\hline $\begin{array}{l}\text { Buen gobierno } \\
\text { y gobernanza. }\end{array}$ & 8,70 & & 14,29 & & 20 & & & & & 50 & & & & & & & & & \\
\hline $\begin{array}{l}\text { Gobierno } \\
\text { central y la } \\
\text { descentrali- } \\
\text { zación de los } \\
\text { gobiernos } \\
\text { locales. }\end{array}$ & 4,35 & 12,50 & & 16,67 & & & & & & & & & & & & 100 & & 100 & \\
\hline Total & 31,50 & 10,96 & 9,59 & 8,22 & 6,85 & 4,11 & 4,11 & 4,11 & 4,11 & 2,74 & 2,74 & 1,37 & 1,37 & 1,37 & 1,37 & 1,37 & 1,37 & 1,37 & 1,37 \\
\hline
\end{tabular}

Fuente: Elaboración propia.

Las investigaciones del GC en el ámbito público también se han situado en otras latitudes geográficas, de una forma más escasa, como son los estudios provenientes de la universidad de Waikato en Nueva Zelanda, la universidad de corea situada en Corea del Sur, la universidad de Aalborg en Dinamarca, la universidad de Barcelona en España, la universidad técnica de Tallin en Estonia, la universidad Tecnológica de Nanyang en Singapur, la universidad del Noroeste en Sudáfrica y la universidad Kristianstad en Suecia (1,37, respectivamente).

Para finalizar, queremos mencionar la relación del número de autores que componen los artículos, es- 


\section{EL GOBIERNO CORPORATIVO EN EL ÁMBITO DEL SECTOR PÚBLICO: UN ESTUDIO BIBLIOMÉTRICO EN LAS REVISTAS UBICADAS EN EL ÁREA DE ADMINISTRACIÓN PÚBLICA}

tos se caracterizan por que usualmente son producidos por dos o más autores $(64,38)$ y en su gran mayoría están elaborados por hombres $(74,36)$. No obstante, algunas investigaciones son producidas fruto del intercambio o colaboraciones entre universidades del ámbito nacional o internacional $(21,92)$. El idioma en el que se encuentran publicados los artículos relacionados con el GC en el sector público es el inglés.

\section{Conclusiones}

El GC cada vez más se está acentuando en el campo del sector público y esto se hace evidente en las publicaciones realizadas en las revistas establecidas en el área de Public Administration, aunque todavía son escasas sus aportaciones, la introducción del concepto del GC al ámbito público es todo un hecho.

En cuanto al estudio bibliométrico realizado se ha podido observar la existencia de diversos estudios científicos que abordan varias áreas de conocimiento en el campo del sector público y estas han girado principalmente en el análisis de sectores como la educación superior, la sanidad o los gobiernos locales. De hecho, la fuerte influencia que ejerce la cultura anglosajona se pone de manifiesto en las diversas revistas que publican esta temática, junto, a las investigaciones realizadas por los países como el Reino Unido, Estados Unidos y Australia, siendo estos países los pioneros en hacer especial hincapié en la implementación y aplicación del GC en el sector público.

Por otra parte, las líneas de investigación del GC en el ámbito público se han centrado en gran parte en temáticas como la reforma y mejoras en la Administración Pública, estilos de gobierno y estructuras de organización e incluso en temas que actualmente tienen una especial repercusión en todo el mundo como es la mejora de la transparencia, la responsabilidad, la rendición de cuentas e incluso la implementación del internet como medio de divulgación de información. Quizás estos elementos junto a la austeridad, el déficit público y la burocracia pueden ser los factores que estén llevando a implementar el GC en el sector público, generando así, una nueva modernización en este campo.
Aunque el GC en el campo del sector público se pone cada vez más sobre el relieve ya bien sea por el interés de los diversos investigadores por contribuir a mejorar las organizaciones del sector público ya que estas se encuentran en un entorno globalizado y altamente competitivo o por la necesidad de la Administración Pública en legitimar sus actuaciones ante los diversos stakeholders. En todo caso, el GC en el sector público se está convirtiendo en una alternativa para los países que se sitúan en las diversas zonas geográficas.

Como futuras líneas de investigación que se pueden plantear en el campo del GC en el ámbito públi$\mathrm{co}$, estas pueden dirigirse a realizar comparaciones con el sector privado y, a su vez, con otros países. También, se puede analizar los avances que realizan los países de la cultura anglosajona pioneros en implementar el GC en el campo del sector público. Asimismo, se puede ampliar el estudio bibliométrico a otras revistas e incorporar otras bases de datos como por ejemplo SCImago Journal \& Country Rank (SJR) de Scopus.

\section{Referencias}

Academic Ranking of World Universities (AMRU) (2015). Shanghai. Recuperado el 16 de julio de 2016, de http://www.shanghairanking.com/es/ARWU2015.html

Adut, D., Holder, A. \& Robin, A. (2013). Predictive versus opportunistic earnings management, executive compensation, and firm performance. Journal of Accounting and Public Policy, 32(3), 126-146.

Bartki, H., Rivard, S. \& Talbot, J. (1988). An Information Systems Keyword Classification Scheme. MIS Quarterly, 12(2), 299-322.

Bordons, M. \& Zulueta, M. (1999). Evaluación de la actividad científica a través de indicadores bibliométricos. Revista Española de Cardiología, (52)10, 1-10.

Bozec, R., Zéghal, D. \& Boujenoui, A. (2004). The effect of the reform of Canadian state-owned enterprises on major corporate governance mechanisms. Australian Journal of Public Administration, 63(2), 79-94.

Braadbaart, O. \& Yusnandarshah, B. (2008). Public Sector Benchmarking: a Survey of Scientific articles, 1990-2005. International Review of Administrative Sciences, 74(3), 421-433.

Cadbury, A. (1992). Informe de comité sobre los aspectos financieros del gobierno corporativo. Recuperado el 16 de junio de 2014, de http://www.ecgi.org/codes/documents/ cadbury.pdf

Carnegie, G. \& Tuck, J. (2010). Understanding the ABC of University Governance. The Australian Journal of Public Administration, 69(4), 431-441. 


\section{JESÚS MAURICIO FLÓREZ-PARRA}

Carver, J. (2010). A case for global governance theory: practitioners avoid it, academics narrow it, the world needs it. Corporate Governance: An International Review, 18(2), 149-157.

Cernat, L. (2004). The emerging European corporate governance model: Anglo-Saxon, Continental, or still the century of diversity? Journal of European Public Policy, 11(1), 147-166.

Christopher, J. (2015). Internal audit Does it enhance governance in the Australian public university sector? Educational Management Administration \& Leadership, 43(6), 954-971.

Clatworthy, A., Mellett, J. \& Peel, J. (2000). Corporate Governance under 'New Public Management': an Exemplification. Corporate Governance, 8(2), 166-176

Cuervo, A. (2002). Corporate Governance Mechanisms: a plea for less code of good governance and more market control. Corporate Governance, 10(2), 84-93

Cuomo, F., Mallin, C. \& Zattoni, A. (2016). Corporate Governance Codes: A Review and Research Agenda. Corporate governance: an international review, 24(3), 222-241.

Davis, J, Schoorman, F. \& Donaldson, L. (1997). Toward a stewardship theory of management. Academy of Management Review, 22(1), 2-47.

De Boer, H., Enders, J. \& Leisyte, L. (2007). Public sector reform in dutch higher education: the organizational transformation of the university. Public Administration, 85(1), 27-46.

Dent, M., Gestel, N. \& Teelken, C. (2007). Symposium on Changing Modes of Governance in Public Sector Organizations: Action and Rhetoric. Public Administration, 85(1), 1-8.

Donaldson, L. \& Davis, J. (1991). Stewardship theory or agency theory: CEO governance and shareholder returns. Australian Journal of Management, 16, 49-64.

Donaldson, T. \& Preston, L. (1995). The stakeholder theory of the corporation: concepts, evidence, and implications. Academy of Management Review, 20(1), 65-91

Durisin, B. \& Puzone, F. (2009). Maturation of corporate governance research, 1993-2007: an assessment. Corporate Governance: An International Review, 17(3), 266-291.

Durisin, B., Calabretta, G. \& Parmeggiani, V. (2010). The Intellectual Structure of Product Innovation Research: A Bibliometric Study of the Journal of Product Innovation Management, 1984-2004. Journal of Product Innovation Management, 27, 437-451.

Ferlie, E., Ashburner, L. \& Fitzgerald, L. (1995). corporate governance and the public sector: some issues and evidence from the NHS. Public Administration, 73(2), 375-392.

Flórez, J. (2013). El gobierno corporativo en el sector público: Un estudio en las universidades públicas españolas. Cuadernos de Administración, 29(50), 142-152.

Flórez, J., López, M. \& López, A. (2014a). El gobierno corporativo de las universidades: Estudio de las cien primeras universidades del ranking de Shanghái. Revista de Educación, 364, 170-196.

Flórez, J., López, M. \& López, A. (2014b). Gobierno corporativo y sector público: un estudio bibliométrico en las principales revistas ISI. Innovar, 24(51), 79-98.

Freeman, E. (1984). Strategic Management: A Stakeholder Approach. Boston: Pitman.
Freeman, E. (1994). The politics of stakeholder theory: some future directions. Business Ethics Quarterly, 4(4), 409-421.

Garand, J. C. (1990). An alternative interpretation of recent polticial science journal evaluations. Political Science and Politics, 23(3), 448-451.

Gómez, P. \& Korine, H. (2005). Democracy and the evolution of corporate governance. Corporate Governance, 13(16), 739-752.

Hacen, M. (2011). Antecedents of organizational innovation the diffusion of new public management into danish local government. Public Administration, 89(2), 285-306.

Hardman, B. (1996). Corporate governance: a practical guide for directors and secretaries. Australian Company Secretary, July, 235-242

Haxhi, I., Ees, H. \& Sorge, A. (2013). A political perspective on business elites and institutional embeddedness in the uk code issuing process. Corporate Governance: An International Review, 21(6), 535-546.

Ho, Chi-K. (2005). Corporate governance and corporate competitiveness: an international analysis. Corporate Governance, 13(2), 211-253.

Hodges, R., Wright, M. \& Keasey, K. (1996). Corporate governance in the public services: concepts and issues. Public Money and Management, 16(2), 7-13.

Hood, C. (1991). A public management for all seasons? Public Administration, 69(1), 3-19.

Hooghiemstra, R. \& Van Ees, H. (2011). Uniformity as response to soft law: evidence from compliance and non compliance with the Dutch corporate governance code. Regulation \& Governance, 5(4), 480-498.

Howard, C. \& Seth-Purdie, R. (2005). Governance Issues for Public Sector Boards. Australian Journal of Public Administration, 64(3), 56-68.

James, O. (2001). Business Models and the Transfer of Businesslike Central Government Agencies. Governance: An International Journal of Policy and Administration, 14(2), 233-252.

Jensen, M. \& Meckling, W. (1976). Theory of the firm: managerial behavior, agency costs and ownership structure. Journal of Financial Economics, 3(4), 305-360.

Journal Citation Reports (JCR) (2015). Institute for Scientific Information. Recuperado el 16 de julio de 2016, de http:// biblioteca.ugr.es/pages/biblioteca_electronica/bases_datos/ journal-citation-reportssocial-sciences

Kellough, J. \& Pitts, D. (2005). Who contributes to public administration review? Examining the charactristics of Authors Who Submit Manuscripts to the Journal. Public administration Review, 65(1), 3-7

La Porta, R., Lopez-De-Silanes, F., Shleifer, A. \& Vishny, R. (2000). Investor protection and corporate governance. Journal of Financial Economics, 58, 3-27.

Lan, Z. \& Anders, K. (2000). A paradigmatic view of contemporary public administration research: an empirical test. Administration and Society, 32(2), 138-165.

Lazonick, W. \& O'Sullivan, M. (2000). Maximizing shareholder value: a new ideology for corporate governance. Economy and Society, 29(1), 13-35. 


\section{EL GOBIERNO CORPORATIVO EN EL ÁMBITO DEL SECTOR PÚBLICO: UN ESTUDIO BIBLIOMÉTRICO EN LAS REVISTAS UBICADAS EN EL ÁREA DE ADMINISTRACIÓN PÚBLICA}

Lee, J. \& Vicente, J. (2005). Bibliometric analysis of human factors (1970-2000): a quantitative description of scientific impact. Human Factors, 47(4), 753-766.

Legge, J. Jr. \& Devore, J. (1987). Measuring Productivity in U.S. Public Administration and public Affair Programs 1981-1985. Administration and Society, 19(2), 147-156

Lokuwaduge, C. \& Armstrong, A. (2015). The impact of governance on the performance of the higher education sector in Australia. Educational Management Administration \& Leadership, 43(5), 811-827.

Luan, Jung-Ch. \& Tang, Je-m. (2007). Where is Independent Director Efficacy? Corporate Governance, 15(4), 636-643.

Nord, J. \& Nord, G. (1995). MIS research: Journal status and analysis. Information and Management, 29(1), 29-42.

Organización para la Cooperación y el Desarrollo Económico (OCDE) (2004). Principios del Gobierno Corporativo. Recuperado el 14 de octubre de 2014, de http://www.oecd. org/daf/ca/corporategovernanceprinciples/37191543.pdf

Organización para la Cooperación y el Desarrollo Económico (OCDE) (2005). Directrices sobre el Gobierno Corporativo de Empresas Públicas. Recuperado el 14 de octubre 2014, de http://www.ecgi.org/codes/documents/oecd_soe_en.pdf

Plümber, T. \& Radaelli, C. (2004). Publish or perish: publications and citations of italian political scientists in international political socience jornals, 1990-2002. Journal of European Public Policy, 11(6), 1112-1127.

Ramos-Rodríguez, A. \& Ruíz-Navarro, J. (2004). Changes in the intellectual structure of strategic management research: a bibliometric study of the strategic management journal, 1980-2000. Strategic Management Journal, 25, 981-1004.
Rhodes, R. (1996). The new governance: governing without government. Political Studies, 44(4), 652-667.

Rodgers, T., Freeman, R., Williams, J. \& Kane, D (2011). Students and the governance of higher education: A UK perspective. Tertiary Education and Management, 17(3), 247-260

Ryan, C. \& Ng, W. (2000). Public sector corporate governance disclosures: an examination of annual reporting practices in Queensland. Australian Journal of Public Administration, 59(2), 11-23.

Ryan, C., Stanley, T. \& Nelson, M. (2002). Accountability disclosures by queensland local government councils: 1997-1999. Financial Accountability \& Management, 18(3), 261-289.

Stewart, J. Asha, F. Shulman, A. \& Ng, C. (2012). Governance disclosure on the internet: the case of australian state government departments. Australian Journal of Public Administration, 71(4), 440-456.

Taylor, M. (2013). Shared governance in the modern university. Higher Education Quarterly, 67(1), 80-94.

Veronesi, G. \& Keasey, K. (2010). NHS boards: knowing the 'what' but not the 'how'. Public Money \& Management, 30(6), 363-370.

Weimer, J. \& Pape, J. (1999). A taxonomy of systems of corporate governance. Corporate Governance, 7(2), 152-166.

Wellens, L. \& Jegers, M. (2011). Beneficiaries' participation in nonprofit organizations: a theory-based approach. Public Money \& Management, 31(3), 175-182.

Whiteoak, J. (1996). Corporate governance-why local government should manage its own agenda. Public Money \& Management, 16(2), 23-29. 